\title{
Reflets
}

Revue d'intervention sociale et communautaire

\section{La prévention des agressions sexuelles auprès des personnes ayant une déficience intellectuelle : vers une plus grande inclusion}

\section{Michèle Diotte}

Volume 21, numéro 1, printemps 2015

URI : https://id.erudit.org/iderudit/1032556ar

DOI : https://doi.org/10.7202/1032556ar

Aller au sommaire du numéro

Éditeur(s)

Reflets, Revue d'intervention sociale et communautaire

ISSN

1203-4576 (imprimé)

1712-8498 (numérique)

Découvrir la revue

Citer cet article

Diotte, M. (2015). La prévention des agressions sexuelles auprès des personnes ayant une déficience intellectuelle : vers une plus grande inclusion. Reflets,

21(1), 215-223. https://doi.org/10.7202/1032556ar d'utilisation que vous pouvez consulter en ligne. 


\section{La prévention des agressions sexuelles auprès des personnes ayant une déficience intellectuelle : vers une plus grande inclusion}

\section{Michèle Diotte}

Centre d'aide et de lutte contre les agressions sexuelles de l'Outaouais (CALAS)

\section{Introduction}

La recherche portant sur la violence sexuelle chez les personnes ayant une déficience intellectuelle (DI) est plutôt limitée (Couture, et collab., 2013). Et les ateliers de prévention des agressions sexuelles s'adressant à cette population sont quasi inexistants. Il y a bien, ici et là, quelques programmes sur la santé sexuelle et sur la violence en général, mais rien de substantiel concernant spécifiquement la violence sexuelle.

Le défi pour le CALAS a donc été de créer de toutes pièces un atelier accessible à ces personnes, c'est-à-dire présenté en termes simples, mais non infantilisants. Souvent comparées à des enfants, les personnes adultes ayant une DI n'ont ni un corps d'enfant, ni une sexualité d'enfant. Le contenu de l'atelier et la façon de le leur transmettre devaient donc s'ajuster à leur réalité d'adultes. 


\section{Violence sexuelle et DI}

\section{Portrait statistique}

Fondées sur des études de type populationnel, les données récentes indiquent qu'à l'âge adulte les personnes ayant une DI sont victimes en plus grand nombre d'agressions sexuelles que la population adulte en général, et même davantage que les autres personnes présentant des incapacités (Couture, et collab., 2013). Selon la recension des écrits réalisée par Mercier (2005), de $70 \%$ à $90 \%$ des femmes ayant une DI sont agressées sexuellement au cours de leur vie, comparativement à $34 \%$ pour le reste de la population féminine (Gouvernement du Québec, 1995).

L'isolement, le manque de connaissance permettant de se prémunir contre diverses formes de violence, la dépendance visà-vis de l'agresseur et la fragilité devant la manipulation font de ces personnes des cibles particulièrement vulnérables (Mercier, 2005). Selon une étude récente menée auprès de 21 personnes de la Mauricie et du Centre-du-Québec ayant une DI, $40 \%$ des agressions sexuelles sont commises par une personne qui doit normalement les soutenir. Quant aux agressions par un pair ayant également une DI, elles représentent $20 \%$ des cas (Couture, et collab., 2013). À la lumière de cette réalité et en réponse aux besoins mis en lumière par ses partenaires œuvrant auprès d'elles, le CALAS de l'Outaouais a cru impératif d'outiller les personnes ayant une DI et d'ainsi réduire leur vulnérabilité à la violence sexuelle.

\section{Cadre théorique : l'intervention féministe intersectionnelle}

Plusieurs éléments expliquent la décision du CALAS de réaliser un outil de prévention des agressions sexuelles s'adressant spécifiquement aux personnes ayant une DI. La perspective féministe intersectionnelle à laquelle adhère le CALAS a permis de développer une analyse de la problématique de l'agression sexuelle dont elles sont victimes. 
L'analyse féministe telle que nous la connaissons met en lumière l'oppression sexiste. Issue du patriarcat, cette forme d'oppression vise universellement les femmes et peut avoir sur elles des conséquences dévastatrices, entre autres, la pauvreté, la violence et la dépendance (Robitaille et Tessier, 2010). Mais l'" approche féministe intersectionnelle " ne se limite pas à l'oppression sexiste; elle implique aussi le chevauchement de plusieurs formes de discriminations. Ainsi, dans une perspective d'intersectionnalité, le sexisme n'est plus la seule forme d'oppression qui est prise en compte (Corbeil et Marchand, 2007).

Depuis déjà plusieurs années, le CALAS est sensible à la discrimination des femmes vivant avec une DI. L'approche féministe intersectionnelle nous rappelle que les modèles d'oppression n'agissent pas indépendamment les uns des autres. Ainsi, les différentes formes d'oppression que sont le sexisme et le capacitisme constituent un système où se chevauchent les discriminations. À ces deux formes d'oppression, peut s'ajouter la discrimination liée, entre autres, à l'orientation sexuelle, à l'appartenance ethnique, à la condition sociale, à l'âge ou à la langue. L'intersection de ces formes d'oppression, de discrimination et d'exclusion est propice à l'exploitation des femmes et favorise la violence sexuelle (Corbeil et Marchand, 2007).

$\mathrm{La}$ violence touche toutes les femmes et les filles. Toutefois, la présence de conditions de précarité ou de multiples discriminations peut être autant de facteurs qui accroissent l'oppression et la vulnérabilité.

C'est dans cette perspective féministe intersectionnelle que nous, au CALAS, avons élaboré notre projet d'atelier. Nous savons que les personnes vivent des formes d'oppression qui se conjuguent, ce qui accroît leur vulnérabilité face à la violence sexuelle à toutes les étapes de leur vie. Il nous apparaissait donc essentiel de les outiller afin qu'elles soient en mesure d'identifier les formes de violence sexuelle qu'elles vivent ou qu'elles ont vécues, afin qu'elles sachent comment y réagir et qu'elles développent des moyens d'autoprotection. 


\section{L'atelier : de la création à la prévention}

La nécessité d'une intervention et d'outils de prévention adaptés a été soulevée à de nombreuses reprises par les différents partenaires œuvrant en DI dans la région de l'Outaouais. Les appuis au projet d'atelier ont donc été nombreux.

Les objectifs du projet sont triples : faire la promotion des rapports égalitaires entre les hommes et les femmes; éliminer la méconnaissance et la tolérance quant à la violence sexuelle vécue par les personnes ayant une DI; outiller ces dernières pour qu'elles se protègent contre la violence sexuelle. L'atelier de prévention des agressions sexuelles et de sensibilisation comporte à cet effet deux volets. Le premier s'adresse aux personnes adolescentes et adultes, hommes et femmes, ayant une DI. Sous forme d'activités interactives, on y définit ce qu'est l'agression sexuelle et on y aborde le droit de vivre en sécurité et sans violence de même que les moyens de protection face à la violence sexuelle. Animé par deux intervenantes du CALAS de l'Outaouais, l'atelier dure deux heures, comprenant au moins une pause, et il est offert à des petits groupes n'excédant pas 10 personnes afin de maximiser les échanges et les apprentissages. Nos expériences d'animation auprès de la clientèle montrent qu'il est pertinent que des intervenantes ou intervenants des organismes visités assistent à l'atelier afin de faciliter la compréhension des participantes ou participants et l'expression de leurs idées. De plus, certains parmi ces derniers ont des besoins spécifiques connus des intervenantes ou des intervenants du milieu visité. Leur présence assure également une continuité en matière d'intervention.

Les exercices présentés dans chacune des activités de l'atelier ont été développés selon la pyramide de mémorisation (Martin et Savary, 1998).

Lorsque j'entends et je vois : je retiens $50 \%$

Lorsque j'entends, je vois, je m'exprime : je retiens $70 \%$ Lorsque j'entends, je vois, je m'exprime et j'agis : je retiens $90 \%$ 
Dans le cadre de l'atelier, les participantes ou participants sont invités à visionner la vidéo Attention! ça peut arriver à tout le monde (Auclair, et collab., 1993), puis à discuter de ce qu'ils ont vu. Nous enchaînons en définissant l'agression sexuelle dans des mots simples et adaptés à leur niveau de langage. Nous présentons alors différents exemples de violence sexuelle, en mettant l'accent sur l'aspect de non-consentement et sur la possibilité que l'agression se fasse avec ou sans attouchements.

Suit l'activité brise-glace La toile de mots qui vise à amener les participantes et les participants eux-mêmes à proposer des exemples d'agression sexuelle et d'émotions ou inquiétudes pouvant être reliées à une situation de violence sexuelle. Cette activité interactive permet également aux personnes d'exprimer leurs émotions.

Comme un des objectifs de l'atelier est la promotion des rapports égalitaires entre les hommes et les femmes, l'activité Est-ce que je connais mes droits? s'avère d'une grande utilité. Nous encourageons les participantes ou participants à identifier les activités qui peuvent être accomplies uniquement par les hommes, uniquement par les femmes ou encore par les personnes de l'un ou de l'autre des deux sexes. Cette activité permet de les sensibiliser au fait que les hommes et les femmes ont des droits identiques : un homme ou une femme peut refuser des avances sexuelles, les personnes des deux sexes ont le droit de dire NON etTOUS ont le droit au respect et à la sécurité.

Dans l'activité Est-ce qu'il m'arrive d'avoir peur d'être agressée? Quoi faire pour me protéger?, nous développons avec les participantes et participants un plan de leur communauté, les amenant à identifier les lieux qu'ils fréquentent. Par la suite, nous leur demandons d'identifier les lieux où ils se sentent bien et ceux où ils ne se sentent pas à l'aise ou pas en sécurité. Cela leur offre des informations précieuses, permettant d'intervenir ultérieurement avec eux. Après avoir réalisé le plan de leur communauté, nous leur demandons d'identifier une personne en qui ils ont confiance et à qui ils peuvent parler s'ils ne se sentent pas en sécurité. Le nom de cette dernière est alors inscrit sur la "carte de secours " qu'ils sont encouragés à garder avec eux comme aide-mémoire. 
L'activité Oui, ça peut arriver à tout le monde d'être agressé sexuellement conclut l'atelier. Dans le cadre de cette activité, nous présentons l'escalier des relations, permettant aux participantes ou aux participants de visualiser les personnes avec qui il est adéquat d'avoir différents types de comportement (par exemple, être nu avec son amoureux) et avec qui cela n'est pas approprié (par exemple, être nu avec le chauffeur d'autobus adapté). Finalement, à l'aide d'illustrations, nous voyons avec eux le concept de consentement et de non-consentement.

Tout au long de l'atelier, des vignettes d'information servent à renforcer l'information transmise. C'est un exemple des outils interactifs utilisés pour susciter la participation. Afin de faciliter l'intégration du contenu de l'atelier, nous avons recours à un soutien visuel important et à beaucoup d'exemples, en répétant constamment les messages clés et en vérifiant fréquemment le niveau de compréhension des participantes ou participants. De plus, lors de la conception de l'atelier, nous avons consulté à plusieurs reprises des intervenantes ou des intervenants œuvrant quotidiennement auprès de personnes ayant une DI. Ils nous ont permis d'adapter certains exercices et de rendre notre atelier le plus accessible possible. Pour nous assurer que ses activités seront comprises par les personnes concernées, nous avons procédé à une validation de l'atelier auprès de trois groupes, ce qui a mené à des ajustements.

Quant au second volet de l'atelier, il s'adresse aux intervenantes et aux intervenants en DI. On y présente le CALAS et son mode de référence et on y aborde la problématique de l'agression sexuelle en lien avec la déficience. D'une durée d'une heure, ce mini atelier renseigne sur la prévalence de la violence sexuelle chez les personnes ayant une DI, ainsi que des conséquences pouvant en découler. Il vise aussi à accroître chez les intervenantes et aux intervenants leurs habiletés à recevoir les dévoilements d'agression sexuelle et à diriger les personnes qui en sont victimes vers les ressources adéquates. 


\section{La prévention et ses défis}

\section{Quelles sont les retombées du projet?}

En 2012-2013, 28 \% des personnes rencontrées dans le cadre $\mathrm{du}$ volet prévention-sensibilisation du CALAS de l'Outaouais avaient une DI (Centre d'aide et de lutte contre les agressions sexuelles de l'Outaouais, 2013). Cela représente 9 ateliers offerts et 74 personnes ayant une DI qui sont désormais mieux outillées pour identifier les agressions sexuelles dont elles pourraient être victimes. De leur côté, pour cette même année, 56 intervenantes et intervenants ont été rencontrés et sensibilisés à la violence sexuelle pouvant être vécue par cette population plus vulnérable.

De plus, nous avons noté une augmentation des références au CALAS de l'Outaouais et des demandes d'aide provenant de personnes ayant une DI. En effet, en 2011-2012 et en 2012-2013, les femmes ayant une DI ont été plus nombreuses que les années antérieures à venir chercher de l'aide chez nous (Centre d'aide et de lutte contre les agressions sexuelles de l'Outaouais, 2013). Le projet a bonifié les collaborations déjà existantes et a permis la création de nouveaux partenariats avec des organismes de la région. Enfin, en plus de briser leur isolement, les ateliers ont offert un lieu sécuritaire aux personnes vivant avec une DI, leur permettant de s'exprimer sur la sexualité et sur la violence sexuelle.

Depuis 2012, le CALAS a offert une conférence à propos du projet dans le cadre du colloque de l'Institut québécois en déficience intellectuelle (IQDI) et a réalisé une présentation lors du congrès du Regroupement québécois des centres d'aide et de lutte contre les agressions à caractère sexuel (RQCALACS). Le projet d'atelier a de plus reçu de belles reconnaissances, tant sur le plan régional que provincial, d'une part avec le Prix David 2013 - Catégorie Sensibilisation décerné par le Regroupement des associations des personnes handicapées de l'Outaouais (RAPHO) et d'autre part avec la Mention d'honneur 2014 - Prévention, Promotion et Protection de la santé et du bien-être décerné par le ministère de la Santé et des Services sociaux du Québec. 


\section{Les défis}

Le principal défi a été de créer de toutes pièces un atelier accessible à la population ciblée, c'est-à-dire offert en termes simples et non infantilisants. Ainsi, nous avons eu à préciser le degré de DI pour lequel l'atelier était conçu. Lors de nos collaborations avec les personnes qui œuvrent auprès de cette population, nous avons été rapidement confrontés à la question suivante : est-ce que l'atelier s'adresse à toutes les personnes ayant une DI ou un trouble envahissant du développement (TED)? Or, il y a différents degrés de DI, allant de léger à profond. Le TED présente également plusieurs particularités.

À la suite des échanges avec les intervenantes ou intervenants travaillant directement avec les personnes ayant une DI et des discussions au sein du CALAS, nous avons décidé de créer un atelier qui serait accessible aux personnes ayant une déficience légère ou modérée. Nous avons constaté que l'atelier ne permet pas d'atteindre les objectifs de sensibilisation avec des personnes ayant une déficience plus sévère. Conséquemment, une population très vulnérable aux agressions sexuelles n'a pas encore accès à une sensibilisation en la matière.

\section{Conclusion}

L'atelier a permis à des personnes ayant une DI de s'exprimer, de poser des questions et de recevoir de l'information utile à propos d'une sexualité saine. Or, nous savons que l'aspect de la sexualité est souvent fort peu abordé, parfois même ignoré, par les intervenantes ou les intervenants. Pourtant, il s'agit d'un enjeu réel. L'atelier leur offre un lieu sécuritaire et sans jugement pour aborder les questions entourant la sexualité et la violence sexuelle.

Nous souhaitons que ce partage de notre pratique suscite l'élaboration d'autres projets de prévention de la violence sexuelle. Socialement, nous devons reconnaître la plus grande vulnérabilité aux agressions sexuelles que vivent les personnes davantage discriminées et agir afin de prévenir cette violence. 


\section{Bibliographie}

AUCLAIR, Hélène, et collab. (1993). Attention! Ça peut arriver à tout le monde, [enregistrement vidéo] Coproduction Centre d'accueil à la Croisée, Centre d'accueil Charleroi, Centres Manouviers, Services de réadaptation du Sud-Ouest, CFCOM de l'Hôpital Des Prairies.

BOURGAULT, France, et Diane MATTE (2010). Ce que nous savons de la violence envers les femmes, Argumentaires pour les 12 jours d'action pour l'élimination de la violence envers les femmes, Comité organisateur des 12 jours d'action pour l'élimination de la violence envers les femmes, $17 \mathrm{p}$.

CENTRE D'AIDE ET DE LUTTE CONTRE LES AGRESSIONS SEXUELLES DE L'OUTAOUAIS (2012). Rapport d'activités du CALAS, [disponible sur demande].

CENTRE D'AIDE ET DE LUTTE CONTRE LES AGRESSIONS SEXUELLES DE L'OUTAOUAIS (2013). Rapport d'activités du CALAS, [disponible sur demande].

CORBEIL, Christine, et Isabelle MARCHAND (2007). L'intervention féministe intersectionnelle : un nouveau cadre d'analyse et d'intervention pour répondre aux besoins pluriels des femmes marginalisées et violentées, Alliance de recherche IREF/Relais-Femmes, Montréal, 21 p.

COUTURE, Germain, et collab. (2013). Les agressions sexuelles subies par les personnes adultes présentant une déficience intellectuelle, Les Collections de l'Institut Universitaire en DI et en TED, 95 p.

GOUVERNEMENT DU QUÉBEC (1995). Les agressions sexuelles STOP, 175 p.

MARTIN, Jean-Paul, et Émilie SAVARY (1998). Intervenir en formation : 12 clés pour préparer, animer, évaluer, Édition Chronique sociale, 221 p.

MER CIER, C. (avril 2005). «La victimisation chez les personnes avec une déficience intellectuelle ", Journal international de victimologie, Tome 3, No 3, p. 209-224.

REGROUPEMENT QUÉBÉCOIS DES CENTRES D'AIDE ET DE LUTTE CONTRE LES AGRESSIONS A CARACTÈRE SEXUEL (2010). La déclaration de principes du RQCALACS, $2 \mathrm{p}$.

ROBITAILLE, Chantal, et Danièle TESSIER (2010). «Trente ans après... Les défis de l'intervention féministe dans les Centres d'aide et de lutte contre les agressions à caractère sexuel (CALACS) ", dans Christine Corbeil et Isabelle Marchand (dires.), L'intervention féministe d'hier à aujourd'hui, p. 49-184, Édition du remue-ménage. 